Original paper

\title{
Characterization of CD326-positive human hepatic stem cells
}

\author{
Eva Schmelzer ${ }^{1}$, Giada Pietrosi², Bruno Gridelli², Jörg Gerlach ${ }^{3}$ \\ 'Department of Surgery, University of Pittsburgh Medical Center, University of Pittsburgh, Pennsylvania, USA \\ ${ }^{2}$ Department for the Treatment and Study of Abdominal Diseases and Abdominal Transplantation, IRCCS-ISMETT, Palermo, Italy \\ ${ }^{3}$ Department of Surgery, Department of Bioengineering, University of Pittsburgh Medical Center, University of Pittsburgh, Pennsylvania, USA
}

\begin{abstract}
Aim of the study: CD326 has been used as a single marker to enrich for hepatic stem cell populations in the liver. However, bile duct epithelium is also positive for CD326, which impedes the selection of pure hepatic stem cell populations. Some markers have been proposed to be co-expressed by hepatic stem cells but these have not been systematically compared. Therefore, we determined the percentages and compared the characteristics of human liver cells expressing potential stem cell surface markers.

Material and methods: We analyzed CD326 expression in human liver tissues from fetal, neonatal, pediatric, and adult stages using immunohistochemistry. In flow cytometry, we quantified fetal liver cells for their co-expression of CD326 with CD56, CD117, CD44, CD90, CD49f, LGR5 and SSEA4. We analyzed the various fractions for their quantitative expression of genes typically associated with progenitors and hepatic lineages.

Results: $12.5 \%$ of cells were positive for CD326; of these, $63.5 \%$ co-expressed CD44. The lowest co-expression percentages were for SSEA4 (2.1\%) and LGR5 (0.7\%). Fractions revealed distinct gene expression patterns. Of all combinations, cells that co-expressed surface CD326 and SSEA4 demonstrated the highest gene expression for the proliferation marker MKi67 and hepatic markers DLK1, AFP and ALB, and were the only fraction negative for the biliary epithelial marker KRT19. Histology of adult and fetal liver showed cells positive for CD326 and SSEA4 but negative for CK19.

Conclusions: CD326-positive cells represent a heterogeneous population, which in combination with SSEA4 potentially distinguishes bile duct epithelium from hepatic stem cells. These findings can help to further classify human hepatic progenitor stages.
\end{abstract}

Key words: CD326, hepatic stem cell, liver, epithelial cell adhesion molecule, EPCAM.

\section{Address for correspondence:}

Dr. Eva Schmelzer, Department of Surgery, University of Pittsburgh Medical Center, University of Pittsburgh, Pennsylvania, USA, e-mail: evaschmelzer@web.de

\section{Introduction}

CD326, also termed epithelial cell adhesion molecule (EPCAM), is a transmembrane glycoprotein. In vivo, it is expressed by multiple normal epithelia including the intrahepatic bile duct epithelium, as well as by carcinomas (for reviews, see [1,2]). Lu et al. [3] demonstrated that CD326 expression on the surface is critical to warrant hepatic specification of endodermal cells during early liver development in zebrafish. In hepatocellular carcinoma (HCC), fractions positive for CD326 have been identified as tumor-initiating subsets [4-7]. In the developing liver, cells that express CD326 can be found in the ductal plate (also called the limiting plate). This is a discrete layer of cells around the portal triads that also expresses cytokeratin 19 (CK19) and neural cell adhesion molecule (NCAM) [8-10]. In the mature liver, the bile duct epithelium and hepatic stem cells are positive for CD326.

Previous work has demonstrated that CD326-positive human hepatic progenitors can be isolated from 
livers of all developmental stages from fetal through adult; furthermore, the hepatic progenitors are CD326positive, but mature hepatocytes are CD326-negative [2, 7, 11-14]. These hepatic progenitors possess longterm expansion and differentiation potential in vitro and in vivo [11, 12, 14-17]. In clinical trials, total suspensions from human fetal liver cells $[18,19]$ as well as CD326-positive cells from human fetal livers selected using magnetic activated cell sorting $[20,21]$ have been successfully transplanted, showing clinical benefits.

Although much progress has been made in the characterization of hepatic stem cells derived from liver (for review, see [22]), using only CD326 for the selection of hepatic stem cell populations does not result in a pure hepatic stem cell population, because the bile duct epithelium is also positive for CD326. There is still a lack of clarity on the expression of further surface markers useful to distinguish hepatic stem cells from bile duct epithelium. Therefore, we aimed to quantify the co-expression of progenitor-associated surface markers with that of CD326, and to further characterize the subpopulations by gene expression analyses.

\section{Material and methods}

\section{Immunohistochemistry}

We investigated CD326 expression in various human developmental stages ranging from fetal to adult. Negative controls included isotype stained sections, and breast cancer tissue sections (BioChain, Hayward, CA) were used as positive controls. Liver tissue pieces were fixed with $4 \%$ para-formaldehyde overnight at $4^{\circ} \mathrm{C}$ and embedded in paraffin. $10 \mu \mathrm{m}$ sections were cut, de-paraffinized with xylene and re-hydrated through decreasing ethanol series. After antigen retrieval (DakoCytomation/Agilent, Santa Clara, CA), hydrogen peroxide block, and avidin/biotin block (Vector Laboratories, Burlingame, CA), sections were incubated with primary mouse immunoglobulin (Ig) G1 anti-CD326 antibody clone Ber-EP4 (Abcam, Cambridge, UK). Negative controls were incubated with mouse IgG1 (DakoCytomation). Secondary antibody staining and horseradish peroxidase amplification was done using the ABC Universal Kit and NovaRed substrate (Vector Laboratories). Cell nuclei were stained with hematoxylin QS (Vector Laboratories). Sections were de-hydrated through increasing ethanol series, fixed with xylene, permanently embedded in VectaMount (Vector Laboratories), and cover-slipped. Images were acquired by bright field microscopy (InvertoskopC), equipped with a digital camera (AxioCam MRc) and software (AxioVision Vs40, V4.2.0.0)
(Zeiss, Oberkochen, Germany). Images were assembled in Adobe Photoshop CS5 Extended Version 12.0 x64 software (Adobe Systems, San Jose, CA).

We also examined CD326, SSEA4, and CK19 coexpression in fetal and adult human liver sections. Acetone-fixed frozen fetal liver sections were purchased from US-Biomax. Sections were blocked overnight at $4^{\circ} \mathrm{C}$ with phosphate-buffered saline (PBS) including $10 \%$ goat serum (Gibco) and 1\% FcR block (Miltenyi Biotec, Bergisch Gladbach, Germany). Primary antibodies mouse IgG1 anti-CD326 clone BerEP4 (Invitrogen/Thermo Fisher Scientific, Waltham, MA), rabbit IgG anti-CK19 polyclonal (NovusBio/ Thermo Fisher Scientific, Waltham, MA), or mouse IgG3 anti-SSEA4 clone MC813-70 (Becton Dickinson, Franklin Lakes, NJ) were applied in blocking buffer for $1 \mathrm{~h}$ at room temperature (RT). Sections were washed three times with PBS, and incubated with secondary antibodies goat anti-mouse IgG1 AF488, goat anti-rabbit IgG AF555, or goat anti-mouse IgG3 AF555 or AF488 in blocking buffer including DAPI for $1 \mathrm{~h}$ at RT. After washing with PBS, the sections were embedded in Aquamount (Polysciences, Warrington, PA) and cover-slipped. Images were taken with a Nikon Eclipse TE300 microscope (Tokyo, Japan) equipped with a ProgRes MF camera and software (Jenoptik, Jena, Germany). Images were assembled in Adobe Photoshop.

\section{Human fetal liver cell isolation}

The isolation of cells from human fetal livers was done as described previously [11] and performed with slight modifications. Human fetal livers of 16 to 20 weeks of gestational age were obtained as anatomical gifts provided by the Allegheny Reproductive Health Center, Pittsburgh, PA. Organs were retrieved from abortions, after informed consent of the donor and approval by the University of Pittsburgh Institutional Review Board. Mechanically disrupted liver tissue pieces were incubated at $37^{\circ} \mathrm{C}$ with $0.6 \mathrm{mg} / \mathrm{ml}$ collagenase type IV, $120 \mathrm{U} / \mathrm{ml}$ DNase, $1 \%$ fatty-acid-free bovine serum albumin, $30 \mathrm{nM}$ selenium (all Sigma-Aldrich, St. Louis, MO), $100 \mathrm{U} / \mathrm{ml}$ penicillin, $100 \mu \mathrm{g} / \mathrm{ml}$ streptomycin, and $0.25 \mu \mathrm{g} / \mathrm{ml}$ Amphotericin B, in RPMI 1640 medium (all Life Technologies, Carlsbad, CA). Cell suspensions were washed twice with RPMI 1640 medium (containing $1 \%$ fatty-acid-free bovine serum albumin, $30 \mathrm{nM}$ selenium, and antibiotic-antimycotic mix) by centrifugation at $300 \mathrm{~g}$, and subsequently filtered through $40 \mu \mathrm{m}$ pore size cell strainers (Becton Dickinson, Bedford, MA). 


\section{Cell numbers and viability}

The total cell numbers and viabilities of freshly isolated cells were determined using a Neubauer chamber and trypan blue (Thermo Fisher Scientific) exclusion in phase contrast microscopy (Zeiss Invertoskop C, Carl Zeiss, Jena, Germany).

\section{Flow cytometry}

Freshly isolated total human fetal liver cells were analyzed using fluorescence-activated cell sorting (FACS). Cells were suspended in $4^{\circ} \mathrm{C}$ buffer containing $0.5 \%$ bovine serum albumin (Sigma-Aldrich), $2 \mathrm{mM}$ di-sodium EDTA (Sigma-Aldrich), antibiotic-antimycotic $(100 \mathrm{U} / \mathrm{ml}$ penicillin, $100 \mu \mathrm{g} / \mathrm{ml}$ streptomycin, and $0.25 \mu \mathrm{g} / \mathrm{ml} \mathrm{Am-}$ photericin B), in PBS without calcium and magnesium (Thermo Fisher Scientific), pH 7.20, and 10\% human FcR blocking reagent (Miltenyi Biotec). Cells were incubated with antibodies or respective isotype controls (Becton Dickinson) for $15 \mathrm{~min}$ at $4^{\circ} \mathrm{C}$ in the dark. Propidium iodide (Sigma-Aldrich, St. Louis, MO) was added for cell viability assessment and potential exclusion of dead cells. Cells were analyzed for their co-expression for CD326 with various surface markers. This panel included mouse IgG1 anti-human CD326-PE (clone HEA125, Miltenyi Biotec), mouse IgG2b anti-human CD44-APCH7 (clone G44-26, BD Biosciences), rat IgG2a anti-human CD49fBV650 (clone GoH3, BD Biosciences), mouse IgG1 antihuman CD56-BB515 (clone B159, BD Biosciences), mouse IgG1 anti-human CD90-BV421 (clone 5E10, BD Biosciences), rat IgG2b anti-human LGR5-AF647 (clone 8F2, BD Biosciences), and mouse IgG3 anti-human SSEA4-BUV395 (clone MC813-70, BD Biosciences). Cells were washed with buffer; $50 \mathrm{U} / \mathrm{ml}$ DNase (Sigma-Aldrich) was included in the final buffer to prevent potential cell clumping. Cells were analyzed and sorted with a FACS Aria II flow cytometer and software (Becton Dickinson), and FlowJo software version 10.4.1 (Tree Star, Ashland, OR). Compensation beads (eBioscience) were used to compensate for fluorochrome spectral overlap. Negative controls included non-stained cells and fluorescence-minus one (FMO) controls. Eight populations were quantified and sorted, consisting of cells expressing $\mathrm{CD} 326^{+}, \mathrm{CD} 326^{+} \mathrm{CD} 117^{+}, \mathrm{CD} 326^{+}$ $\mathrm{CD}_{4} 4^{+}, \mathrm{CD} 326^{+} \mathrm{CD} 49 \mathrm{f}^{+}, \mathrm{CD} 326^{+} \mathrm{CD} 56^{+}, \mathrm{CD} 326^{+} \mathrm{CD} 90^{+}$, $\mathrm{CD}_{22} 6^{+} \mathrm{LGR}^{+}$, and CD $326^{+} \mathrm{SSEA} 4^{+}$. Subsequent co-expression combinations were also quantified.

\section{Gene expression analyses}

Freshly isolated total fetal liver cells and sorted fetal liver cells were lysed directly with RLT buffer, and RNA was isolated using shredder and isolation columns (RNeasy-mini kit, Qiagen, Valencia, CA), including DNA digestion by DNase treatment on columns. Concentrations of nucleic acids were determined fluorometrically using Quant-iT Assay kits and a Qubit fluorometer (Thermo Fisher Scientific). RNA was reverse transcribed to CDNA with the High-Capacity cDNA Reverse Transcription Kit (Applied Biosystems, Carlsbad, CA). Gene expression was analyzed using real-time PCR using a StepOnePlus system and software version 2.0, and pre-designed TaqMan probe and primer assay mixes With Gene Expression Master Mix (Applied Biosystems). $\beta$-actin served as a housekeeping gene for internal normalization. TaqMan assay mixes were: ACTB (actin $\beta$ ), AFP ( $\alpha$-fetoprotein), ALB (albumin), DLK1 (delta-like 1 homolog), EPCAM (epithelial cell adhesion molecule; CD326), KRT19 (keratin 19, type 1; cytokeratin 19), HNF4a (hepatocyte nuclear factor 4a), LGR5 (leucine-rich repeat containing $\mathrm{G}$ protein-coupled receptor 5), MKI67 (marker of proliferation Ki-67), POU5F1 (POU class 5 homeobox 1; Oct4), and THY1 (Thy-1 cell surface antigen; CD90). Expression was quantified using the ddCt method. Freshly isolated total human fetal liver cells served as positive controls; no template (water) was used as a negative control. Each of the four biological samples was analyzed with two technical repeats.

\section{Statistics}

Data are given as means from $n$ biological repeats \pm standard deviation. Student's $t$-test was used to analyze statistically significant differences. Asterisks indicate $p$-values: ${ }^{\star} p \leq 0.05,{ }^{* *} p \leq 0.01,{ }^{* * *} p \leq 0.001$.

\section{Results}

\section{CD326 expression in human liver tissues of various developmental stages}

We investigated CD326 expression in normal human liver tissues from various developmental stages ranging from fetal, neonatal, adolescent, younger adult, to older adult (Fig. 1). In fetal liver, progenitors of the ductal plates stained positive for CD326; bile ducts could be observed, which were also always positive for CD326. The vast majority of parenchymal hepatoblasts did not stain positive for CD326. In neonatal to adult tissues, CD326-positive cells were bile duct epithelium; occasionally, CD326-positive cells in the ductal plate could be observed.

\section{Cell yields and viabilities}

Isolations from 16 to 20 weeks gestational livers yielded $1.33 \times 10^{9} \pm 0.58 \times 10^{9}$ total human fetal liver 

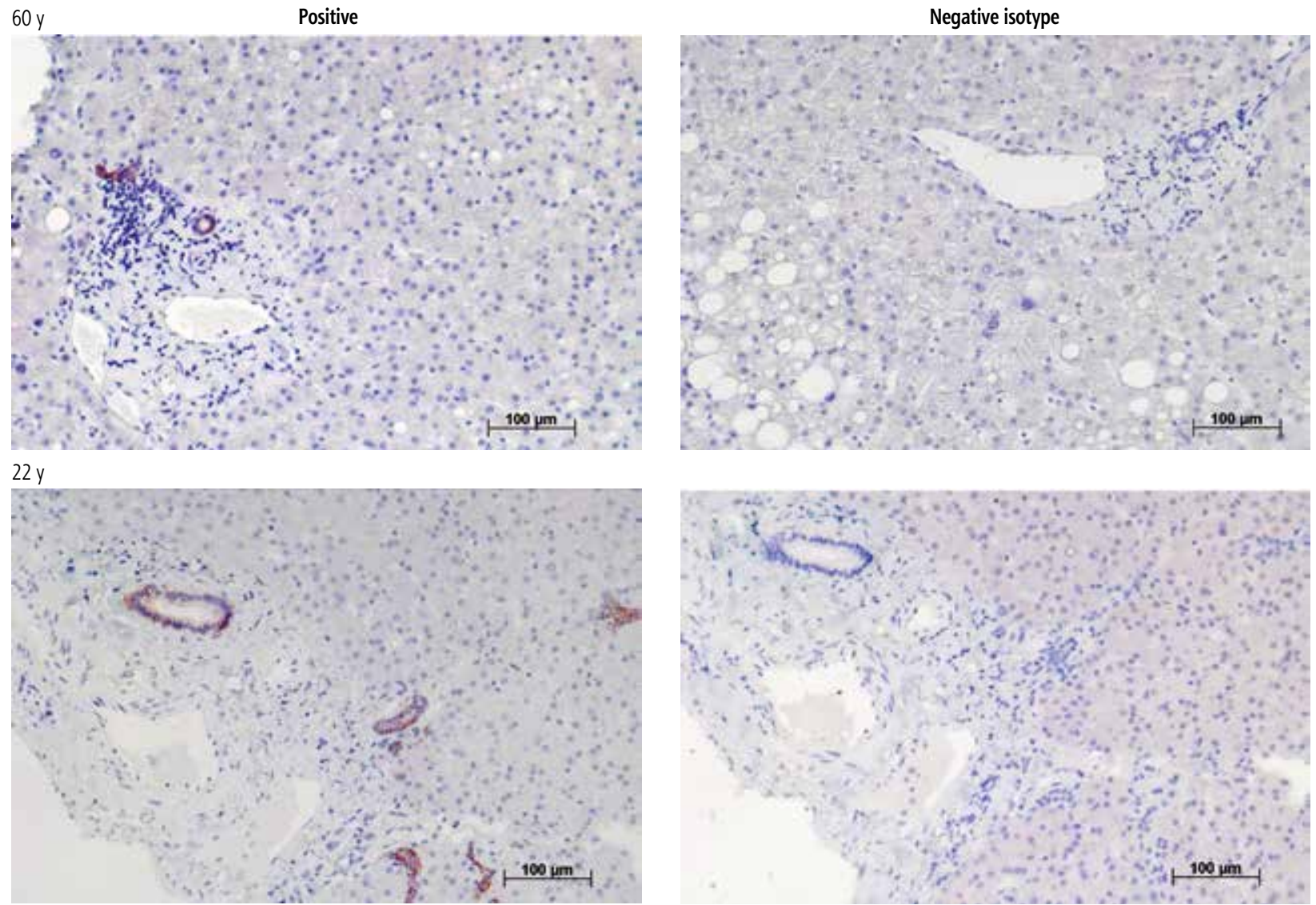

$15 y$
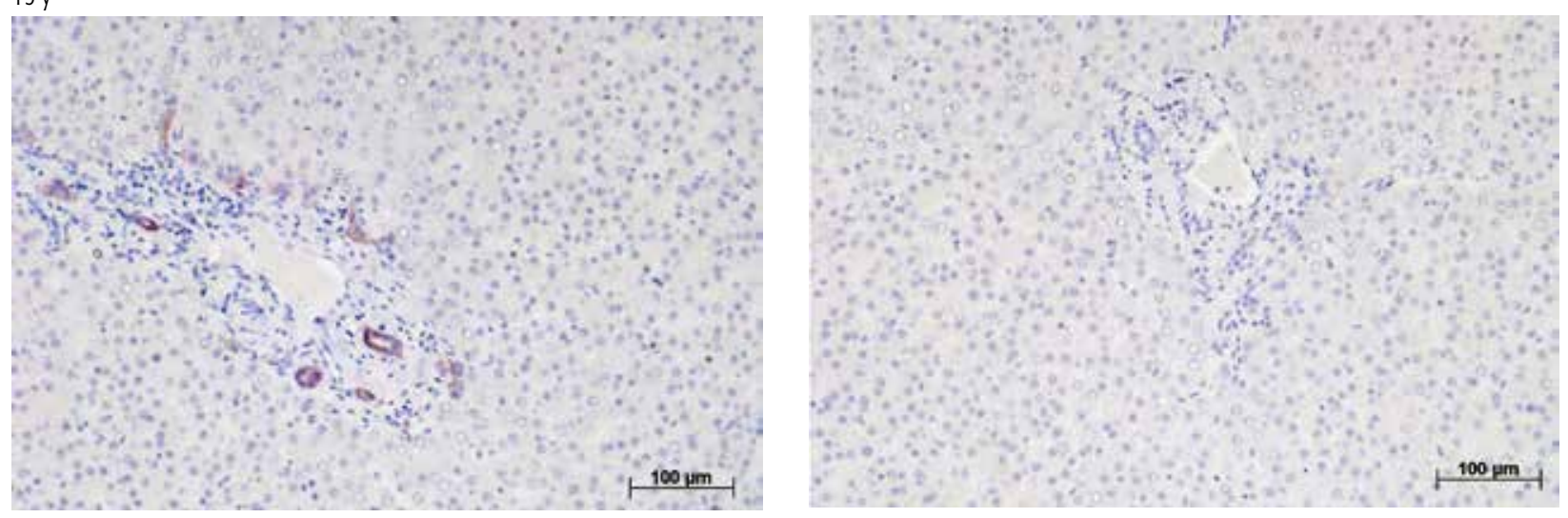

$9 y$
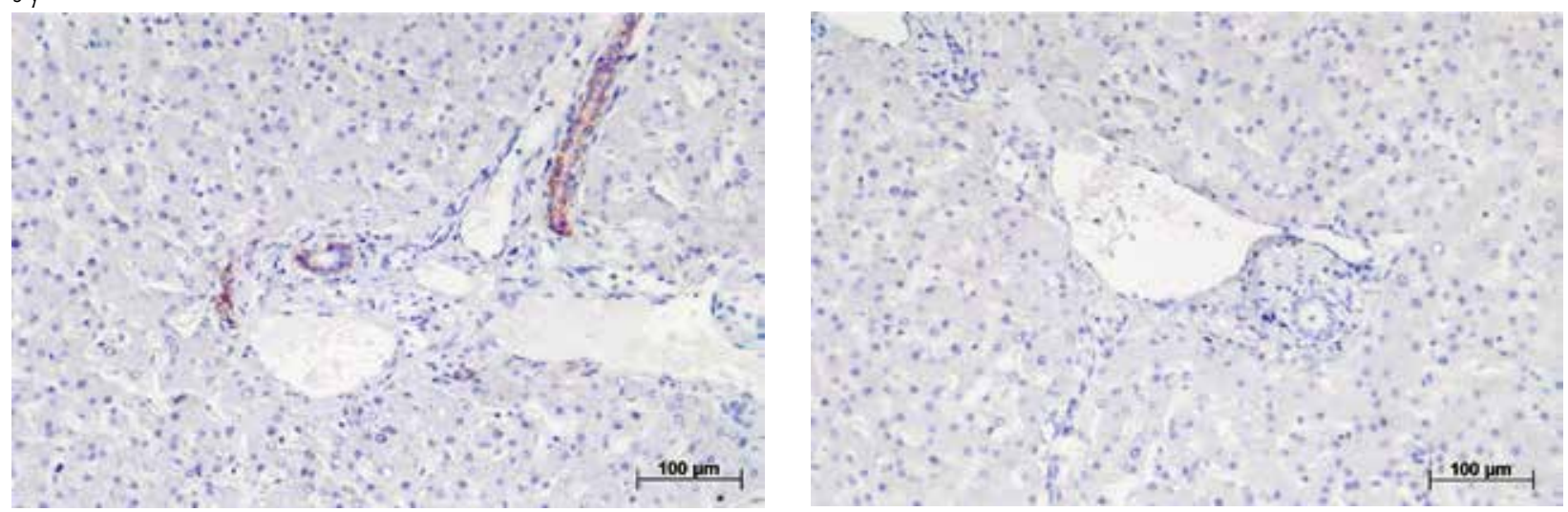

Fig. 1. Immunohistochemistry of human liver sections. Paraffin-embedded formaldehyde-fixed sections of human liver tissues from various developmental stages were stained for CD326 (red), cell nuclei were stained with hematoxylin (blue); A) positive stain, B) isotype negative control. Abbreviations of developmental stages: $w$ - fetal gestational week, $m$ - postnatal month, $y$ - pediatric to adult year 

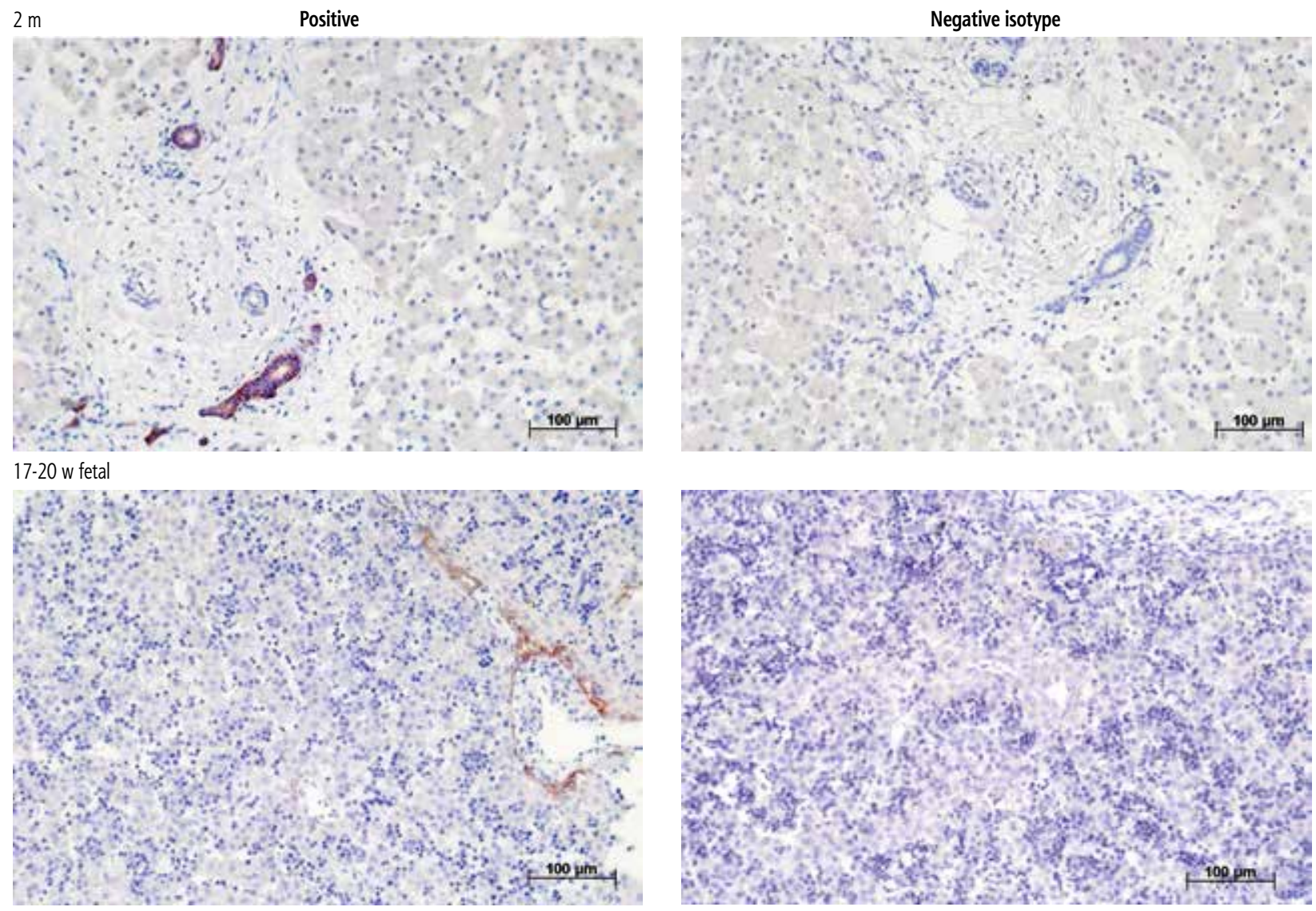

Fig. 1. Cont; A) positive stain, B) isotype negative control. Abbreviations of developmental stages: $w$ - fetal gestational week, $m$ - postnatal month, $y$ - pediatric to adult year

cells by average. Due to variations in gestational age, and the nature of the specimen retrieval procedure, in which tissues are usually not obtained as a complete, whole organ, some variation in the total cell yield resulted. Freshly isolated cells had viabilities of $96.8 \%$ $\pm 1.5 \%$ as determined by trypan blue exclusion assay.

\section{Flow cytometry}

We analyzed and sorted total human fetal liver cell suspensions using FACS. Cell viabilities after the staining procedure during flow cytometry were $96.7 \%$ on average as determined by propidium iodide staining, which was equal to viabilities of freshly isolated cells. We investigated the co-expression profiles of the surface marker CD326 with other potential hepatic stem-cell-associated markers, namely CD44, CD49f, CD56, CD90, CD117, LGR5, and SSEA4 (Table 1). From the total liver cell suspension, we found $12.5 \%$ of cells to be positive for CD326. $63.5 \%$ of $\mathrm{CD} 326^{+}$cells were also co-expressing CD44, resulting in overall $8.1 \%$ of total cells being $\mathrm{CD} 326^{+} \mathrm{CD} 44^{+} .26 .6 \%$ of $\mathrm{CD} 326$ positive cells co-expressed CD117, and $11.2 \%$ were CD90 positive. Minor but detectable fractions of the $\mathrm{CD} 326$ positive cells expressed CD56, CD49f, SSEA4, or LGR5 $(4.2 \%, 3.5 \%, 2.1 \%$, or $0.68 \%$, respectively). Subsequent co-expression analyses of the third level revealed further heterogeneity of the respective cell populations.

In order to further characterize the prospective progenitor cells, eight populations were sorted and collected, consisting of a total of CD326 positive cells as wells as cells co-expressing CD326 with CD44, CD49f, CD56, CD90, CD117, LGR5, or SSEA4, respectively. Subsequently, these eight sorted populations of cells were subject to gene expression analyses.

\section{Gene expression analyses}

We investigated the gene expression profiles of the FACS-sorted, eight hepatic stem cell populations (Fig. 2). In particular, we examined the expression of genes characteristic for specific developmental stages, including EPCAM and cytokeratin 19 (KRT19) for hepatic epithelial stem cells and bile duct epithelium, AFP and DLK1 for hepatoblasts, ALB and HNF4a for hepatocytes, LGR5 and POU5F1 (POU class 5 homeobox 1; Oct4) as typical genes expressed by stem cells, MKI67 (marker of proliferation Ki-67) expressed by 
Table 1. Quantitative flow cytometry analyses of surface marker expression. Freshly isolated total human fetal liver cells were analyzed for their expression of surface markers potentially associated with hepatic stem cells. Dead cells, debris and doublets were excluded by propidium iodide dye stain and applying forward versus side scatter gates (fsc/ssc). A) Percentages of cells of the total live forward vs. side scatter (fsc/ssc) population positive for each single surface marker. B) Percentages of cells within the CD326+ fraction co-expressing subsequently a second and third surface marker, given as percentages of respective parent or of total live fsc/ssc fraction. Data are given from $n=4$ biological repeats \pm standard deviation

A

\begin{tabular}{lcccccccc}
\hline Surface marker & CD44 & CD326 & CD117 & CD90 & CD56 & CD49f & SSEA4 & LGR5 \\
\hline From total live & 20.6 & 12.5 & 4.0 & 2.1 & 0.82 & 0.73 & 0.34 & 0.12 \\
fsc/ssc (\%) & \pm 9.5 & \pm 2.8 & \pm 1.7 & \pm 0.7 & \pm 0.34 & \pm 0.59 & \pm 0.22 & \pm 0.42 \\
\hline
\end{tabular}

B

\begin{tabular}{|c|c|c|c|c|}
\hline $\begin{array}{l}\text { Second surface marker } \\
\text { from CD326 (\%) }\end{array}$ & From parent (\%) & From total live fsc/ssc (\%) & Third surface marker & From parent (\%) \\
\hline \multirow[t]{6}{*}{ CD44 } & $63.5 \pm 14.9$ & $8.1 \pm 2.6$ & CD117 & $36.6 \pm 11.2$ \\
\hline & & & CD90 & $8.8 \pm 4.7$ \\
\hline & & & CD56 & $2.3 \pm 0.8$ \\
\hline & & & SSEA4 & $1.9 \pm 1.6$ \\
\hline & & & CD49f & $1.7 \pm 2.1$ \\
\hline & & & LGR5 & $0.9 \pm 0.8$ \\
\hline \multirow[t]{6}{*}{ CD117 } & $26.6 \pm 10.6$ & $3.2 \pm 1.3$ & CD44 & $94.4 \pm 2.2$ \\
\hline & & & CD90 & $8.3 \pm 4.7$ \\
\hline & & & CD56 & $3.7 \pm 2.0$ \\
\hline & & & SSEA4 & $2.4 \pm 1.8$ \\
\hline & & & CD49f & $1.9 \pm 2.2$ \\
\hline & & & LGR5 & $1.6 \pm 1.1$ \\
\hline \multirow[t]{6}{*}{ CD90 } & $11.2 \pm 2.6$ & $1.4 \pm 0.7$ & CD44 & $38.0 \pm 14.5$ \\
\hline & & & CD56 & $41.0 \pm 28.6$ \\
\hline & & & CD117 & $16.9 \pm 8.8$ \\
\hline & & & CD49f & $12.4 \pm 13.4$ \\
\hline & & & SSEA4 & $9.7 \pm 3.9$ \\
\hline & & & LGR5 & $5.2 \pm 3.6$ \\
\hline \multirow[t]{6}{*}{ CD56 } & $4.2 \pm 1.7$ & $0.52 \pm 0.21$ & CD90 & $90.7 \pm 6.1$ \\
\hline & & & CD44 & $42.1 \pm 4.8$ \\
\hline & & & CD49f & $25.6 \pm 25.2$ \\
\hline & & & CD117 & $21.8 \pm 6.7$ \\
\hline & & & SSEA4 & $14.2 \pm 5.0$ \\
\hline & & & LGR5 & $10.9 \pm 6.1$ \\
\hline \multirow[t]{6}{*}{ CD49f } & $3.5 \pm 3.2$ & $0.44 \pm 0.40$ & CD90 & $55.8 \pm 15.3$ \\
\hline & & & CD56 & $30.1 \pm 17.4$ \\
\hline & & & CD44 & $27.9 \pm 11.5$ \\
\hline & & & CD117 & $12.8 \pm 9.6$ \\
\hline & & & SSEA4 & $16.0 \pm 7.4$ \\
\hline & & & LGR5 & $7.8 \pm 4.7$ \\
\hline \multirow[t]{6}{*}{ SSEA4 } & $2.1 \pm 1.2$ & $0.26 \pm 0.15$ & CD90 & $66.9 \pm 13.1$ \\
\hline & & & CD44 & $55.6 \pm 26.7$ \\
\hline & & & CD56 & $32.0 \pm 18.3$ \\
\hline & & & CD117 & $29.8 \pm 17.1$ \\
\hline & & & CD49f & $21.4 \pm 11.0$ \\
\hline & & & LGR5 & $9.1 \pm 8.5$ \\
\hline \multirow[t]{6}{*}{ LGR5 } & $0.68 \pm 0.42$ & $0.092 \pm 0.070$ & CD44 & $86.2 \pm 7.0$ \\
\hline & & & CD90 & $75.1 \pm 8.7$ \\
\hline & & & CD56 & $61.8 \pm 21.4$ \\
\hline & & & CD117 & $60.4 \pm 18.2$ \\
\hline & & & CD49f & $35.9 \pm 22.7$ \\
\hline & & & SSEA4 & $22.2 \pm 4.0$ \\
\hline
\end{tabular}


proliferating cells, and THY1 (Thy- 1 cell surface antigen; CD90), which is expressed by mesenchymal and mesendodermal progenitors.

Total freshly isolated human fetal liver cells were used as a normalizer for gene expression (set as $100 \%)$. Overall, with most populations we could observe distinct expression patterns associated with the differently sorted populations. $\mathrm{CD} 326^{+} \mathrm{CD} 117^{+}$and $\mathrm{CD} 326^{+} \mathrm{CD} 44^{+}$populations demonstrated similar patterns of analyzed genes. These were also similar to those of the total CD326 fraction. Sorted cells demonstrated highly different levels of EPCAM expression. The total CD326 fraction and cells that co-expressed either CD117, CD44, or SSEA4 had higher EPCAM expression than the total human fetal liver cell fraction. The $\mathrm{CD} 326^{+} \mathrm{LGR} 5^{+}$population demonstrated the lowest EPCAM gene expression.

Interestingly, cells that co-exhibited CD326 and SSEA4 on their surface had the strongest gene expression for the proliferation marker MKi67 and hepatoblast markers DLK1, AFP and ALB, but it was the only population that was negative for the biliary epithelial marker KRT19, thus potentially distinguishing bile duct epithelium from hepatic stem cells.

\section{Co-expression of CD326 with SSEA4 or CK19 in human liver tissues}

Based on the results of our gene expression analyses, we studied the co-expression of CD326 with
CK19, CK19 with SSEA4, and CD326 with SSEA4 in fetal (Fig. 3) and adult (Fig. 4) liver tissues, because co-expression studies with the intracellular CK19 could not be performed in flow cytometry. As expected from FACS and gene expression studies, we found that most fetal cells positive for CD326 also expressed CK19 (Fig. 3A), but occasionally a few cells that were positive for $\mathrm{CD} 326$ but negative for CK19 could be observed (for example, visible in the lower right corner of the frame). A few cells that were positive for SSEA4 could be observed (Fig. 3B, C). These were located at the limiting plate, the anticipated location of hepatic stem cells in the fetal liver. Furthermore, these were mostly negative for CK19, and CK19-positive cells were mostly negative for SSEA4 (Fig. 3B). Most of the SSEA4-positive cells were also positive for CD326 (Fig. 3C), but some SSEA4-positive cells could be observed that were not positive for CD326. In adult liver (Fig. 4), we found that, compared to fetal liver, fewer of the CK19-positive cells were also positive for CD326. Similar to fetal liver, very few cells that were positive for SSEA4 could be observed (Fig. 4B, C). Some of these few cells were positive for CK19 and some negative, while most of the CK19-positive cells were negative for SSEA4 (Fig. 4B).

\section{Discussion}

CD326 is expressed on the cell surface of normal epithelia of the body [2] as well as on several cancers

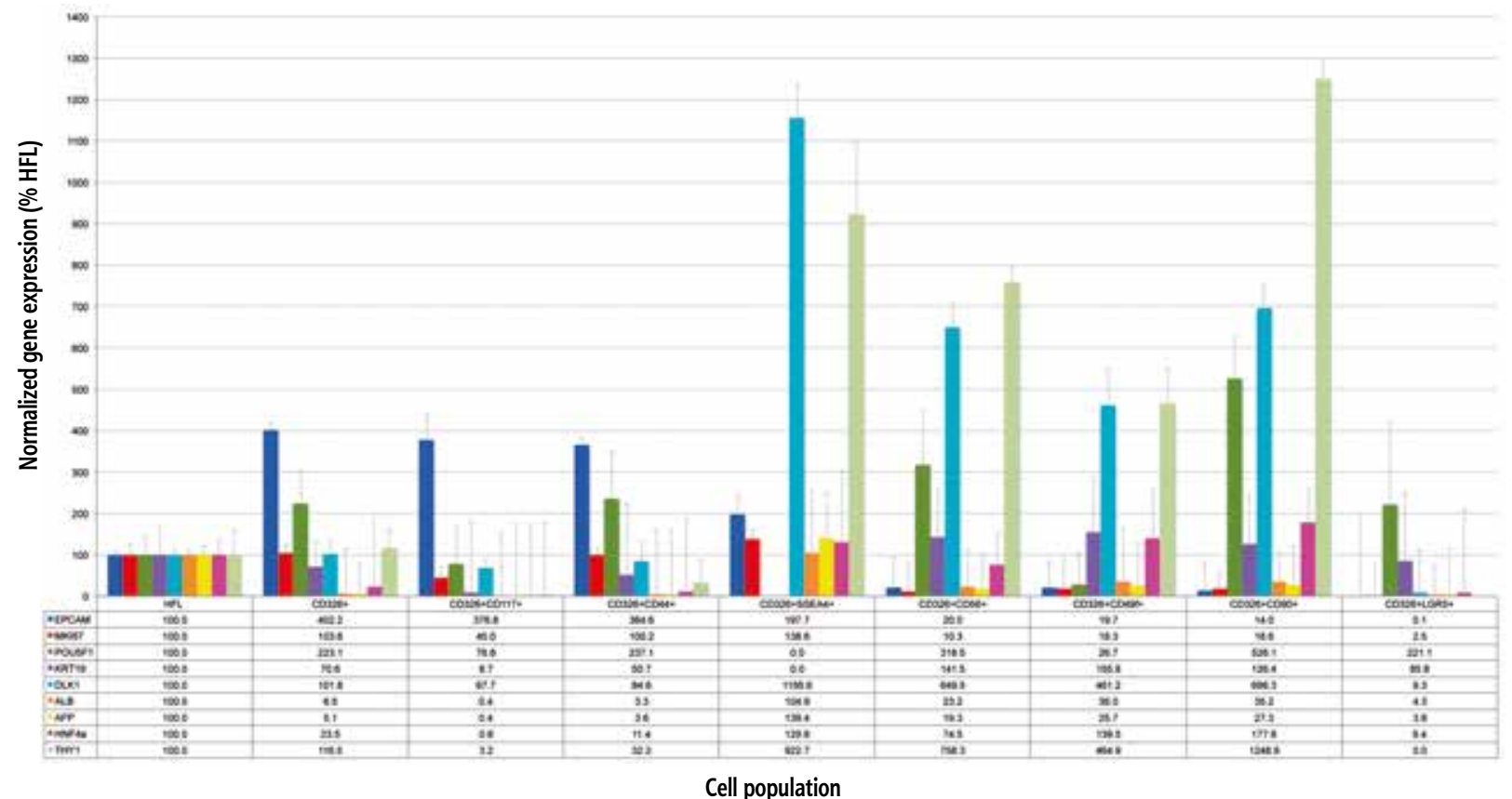

Fig. 2. Gene expression analyses. Total human fetal liver (HFL) cells and sorted cell fractions were analyzed for their gene expression of EPCAM, MIK67, POU5F1, KRT19, DLK1, ALB, AFP, HNF4a, and THY1 
A

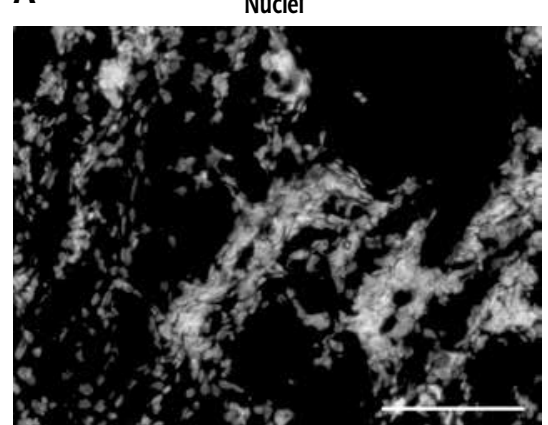

B

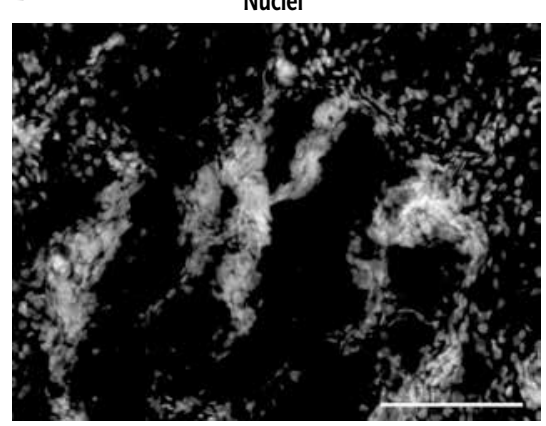

Nuclei

C

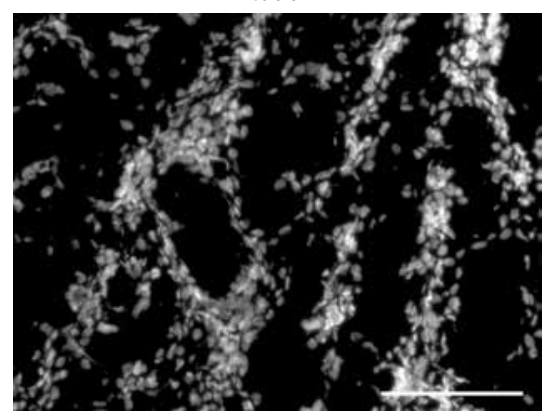

CK19

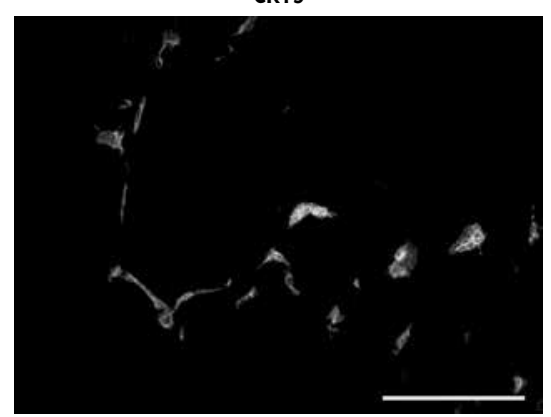

CK19

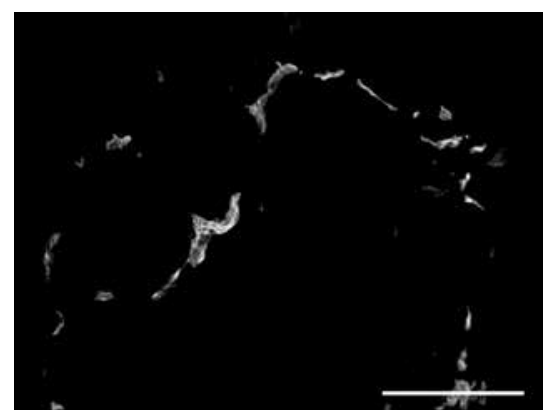

CD326

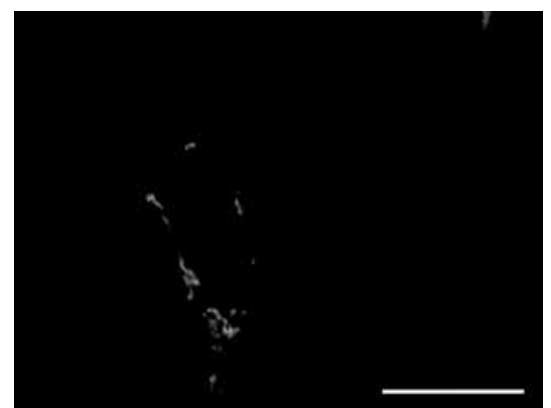

CD326

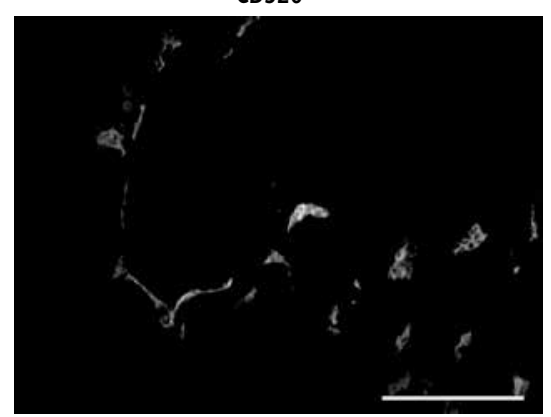

SSEA4

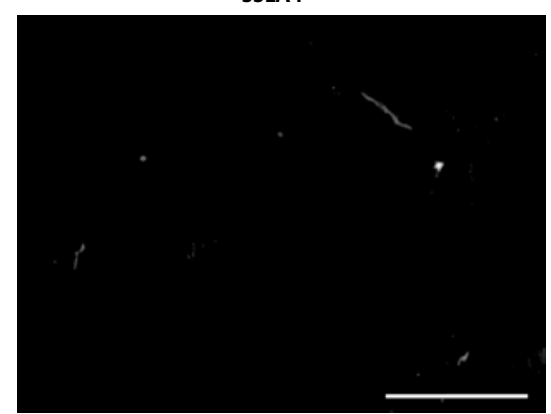

SSEA4

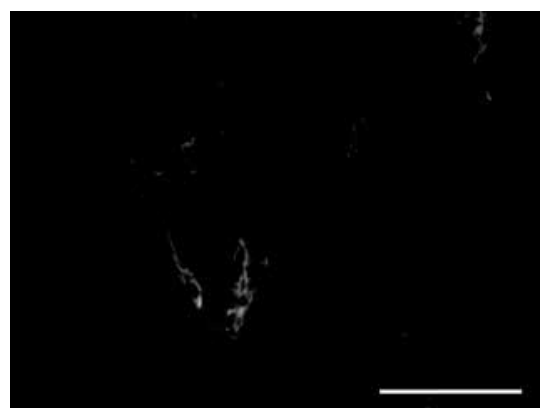

Fig. 3. Immunofluorescence of human fetal liver sections. Frozen fetal liver tissue sections were stained for CK19, CD326, SSEA4 and cell nuclei with DAPI. A, B and C represent same sections. Scale bars: $150 \mu \mathrm{m}$

$[4-6,23,24]$. CD326 regulates both cell migration and cell adhesion, favors tissue polarization and regulates intracellular signaling [25]. The role and the expression of CD326 in liver regeneration and liver injury is still a topic of debate. Signaling of the intracellular domain of CD326 has been shown to be an important mechanism to induce proliferation in cancer cells [26] but not in normal CD326-positive cells of the liver [27]. In the healthy liver, the majority of cells positive for CD326 are bile duct epithelial cells, as shown previously [11, 12, 14-17], and as confirmed in Figure 1 for various developmental stages of the human liver. Although hepatic stem cells are also positive for CD326, the selection for CD326 results in an enriched but not pure hepatic stem cell population.

Some studies have revealed further surface markers that potentially are positively or negatively associated with hepatic stem cells. A major drawback, however, is that most studies cultured the total human fetal liver cell population and associated retrospectively the prospective cellular characteristics, i.e. prospective hepatic stem cells were not selected initially from the total liver cell population. This approach inadvertently will include the possibility of cellular changes in culture, does not represent a purified stem cell population and may not necessarily resemble in vivo development. However, this approach still provides the means of possibly finding discriminative surface markers and other characteristics of hepatic stem cells.

Studies from Dan et al. [14] characterized small cell types that emerged from total human fetal liver cell cultures, which were cultured for longer term, as described previously [28]. These cells were positive for CD34, CD90, c-kit, EPCAM, c-met, SSEA4, CK18, CK19, $\mathrm{CD} 44 \mathrm{~h}$, and vimentin, but negative for albumin and AFP. Previous studies $[11,12]$ have shown that human 
A

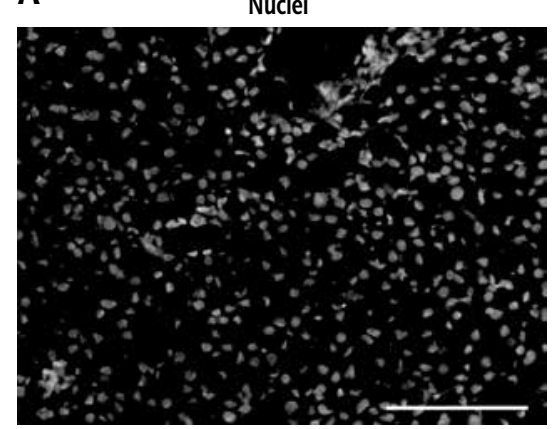

B

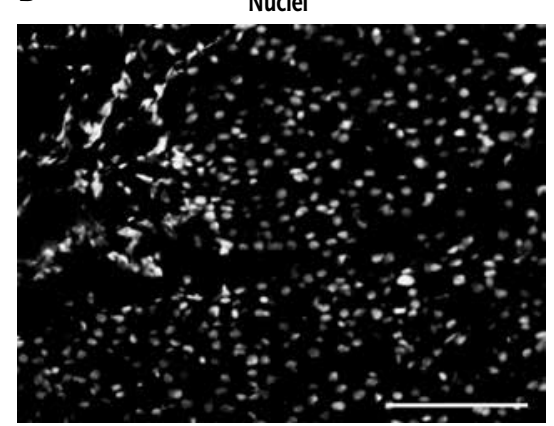

C

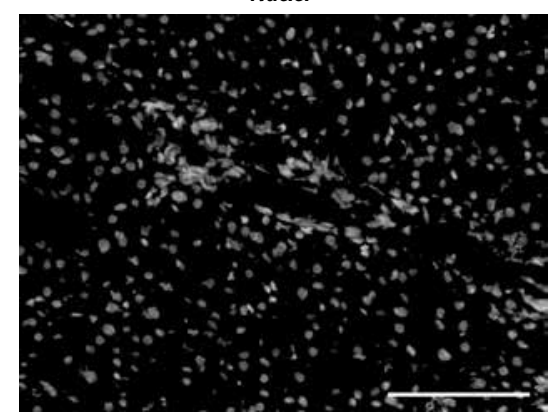

CK19

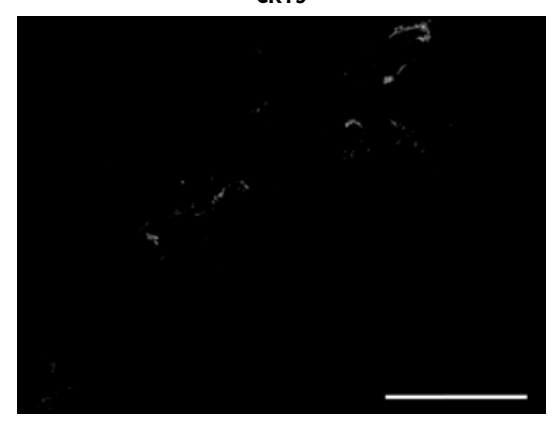

CK19

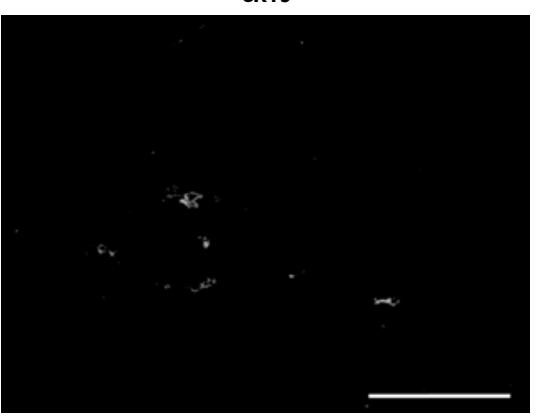

CD326

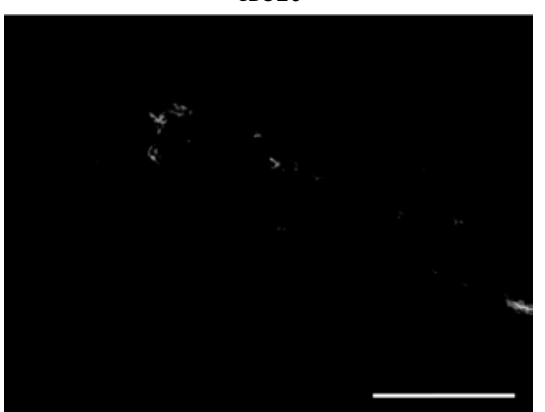

CD326

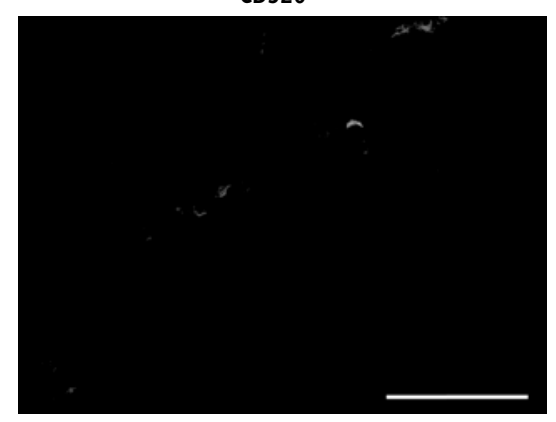

SSEA4

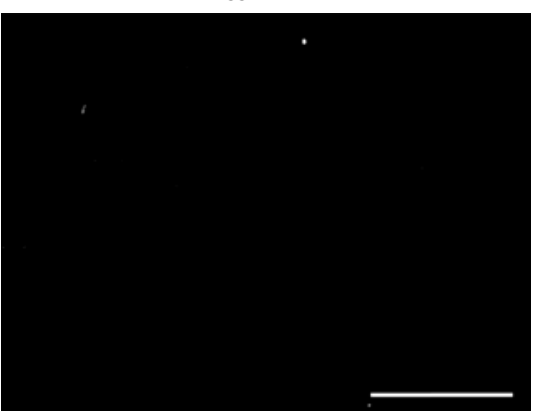

SSEA4

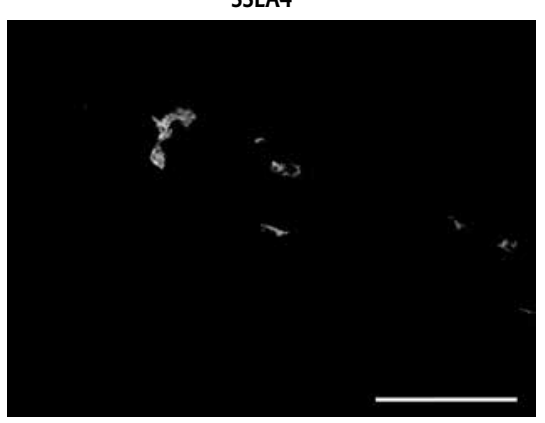

Fig. 4. Immunofluorescence of human adult liver sections. Frozen adult liver tissue sections were stained for CK19, CD326, SSEA4 and cell nuclei with DAPI. A, B and C represent same sections. Scale bars: $150 \mu \mathrm{m}$

fetal liver cell culture under selective conditions supports expansion and maintenance of hepatic stem cells, which were, among other markers, positive for CD326. Fomin et al. [17] found that cells isolated from human fetal liver positive for CD326 but negative for CD14 were a mixture of parenchymal cells, cholangiocytes, and hepatoblasts. In culture, cells gave rise to cells positive for albumin, cells positive for cytokeratin-19, and cells expressing both proteins. The CD326-positive CD14-negative cells demonstrated, among others, gene expression for ALB, AFP, and CK19. Huch et al. [15] performed cultures of total cells from human adult liver biopsies, followed by selective cloning. CD326-positive organoids expressed LGR5, CK19 and HNF4 but no ALB; in immunocytochemistry, only a subset of CD326-positive cells was also positive for LGR5. We found in flow cytometry that the percentage of freshly isolated, non-cultured LGR5-positive cells from total fetal liver was considerably low (on average $0.12 \%$ ); likewise, the percentage of CD326-positive cells (on average $12.5 \%$ of total cells) that also co-expressed LGR5 was extremely low (on average only $0.68 \%$ of CD326-positive cells). Lgr5 has been considered a marker of liver progenitor cells following damage [29], which would explain its low expression in normal liver. Cardinale et al. [21] found higher percentages of Lgr5 co-expression in CD326-positive cells. Two human fetal liver were sorted with magnetic beads for CD326. Flow cytometry showed that from these two total fetal livers, about $25.5 \%$ of the total cells were positive for CD326, and nearly all of them co-expressed Lgr5 (on average $24.6 \%$ of the total).

\section{Acknowledgments}

This study was financially supported by the University of Pittsburgh Medical Center. We thank the staff 
of the Allegheny Reproductive Health Center and the Flow Cytometry Core Facility of the McGowan Institute for Regenerative Medicine for their continuous outstanding support.

\section{Disclosure}

The authors declare no conflict of interest.

\section{References}

1. Dolle L, Theise ND, Schmelzer E, et al. EpCAM and the biology of hepatic stem/progenitor cells. Am J Physiol Gastrointest Liver Physiol 2015; 308: G233-250.

2. Schmelzer E, Reid LM. EpCAM expression in normal, non-pathological tissues. Front Biosci 2008; 13: 3096-3100.

3. Lu H, Ma J, Yang Y, et al. EpCAM is an endoderm-specific Wnt derepressor that licenses hepatic development. Dev Cell 2013; 24: 543-553.

4. Yamashita T, Budhu A, Forgues M, et al. Activation of hepatic stem cell marker EpCAM by Wnt-beta-catenin signaling in hepatocellular carcinoma. Cancer Res 2007; 67: 10831-10839.

5. Yamashita T, Forgues M, Wang W, et al. EpCAM and alpha-fetoprotein expression defines novel prognostic subtypes of hepatocellular carcinoma. Cancer Res 2008; 68: 1451-1461.

6. Yamashita T, Ji J, Budhu A, et al. EpCAM-positive hepatocellular carcinoma cells are tumor-initiating cells with stem/progenitor cell features. Gastroenterology 2009; 136: 1012-1024.

7. de Boer CJ, van Krieken JH, Janssen-van Rhijn CM, et al. Expression of Ep-CAM in normal, regenerating, metaplastic, and neoplastic liver. J Pathol 1999; 188: 201-206.

8. Ruebner BH, Blankenberg TA, Burrows DA, et al. Development and transformation of the ductal plate in the developing human liver. Pediatr Pathol 1990; 10: 55-68.

9. Balzar M, Winter MJ, deBoer CJ, et al. The biology of the 17-1A antigen (Ep-CAM). J Mol Med 1999; 77: 6999-6712.

10. Van Den Heuvel M, Sloof M, Visser L, et al. Expression of anti-OV6 antibody and anti-N-CAM antibody along the biliary line of normal and diseased human livers. Hepatology 2001; 33 : 1387-1393.

11. Schmelzer E, Wauthier E, Reid LM. The phenotypes of pluripotent human hepatic progenitors. Stem Cells 2006; 24: 1852 1858.

12. Schmelzer E, Zhang L, Bruce A, et al. Human hepatic stem cells from fetal and postnatal donors. J Exp Med 2007; 204: 1973-1987.

13. Yoon SM, Gerasimidou D, Kuwahara R, et al. Epithelial cell adhesion molecule (EpCAM) marks hepatocytes newly derived from stem/progenitor cells in humans. Hepatology 2011; 53: 964-973.

14. Dan YY, Riehle KJ, Lazaro C, et al. Isolation of multipotent progenitor cells from human fetal liver capable of differentiating into liver and mesenchymal lineages. Proc Natl Acad Sci U S A 2006; 103: 9912-9917.

15. Huch M, Gehart H, van Boxtel R, et al. Long-term culture of genome-stable bipotent stem cells from adult human liver. Cell 2015; 160: 299-312.

16. Fomin ME, Beyer AI, Muench MO. Human fetal liver cultures support multiple cell lineages that can engraft immunodeficient mice. Open Biol 2017; 7: 170108.

17. Fomin ME, Tai LK, Barcena A, et al. Coexpression of CD14 and CD326 discriminate hepatic precursors in the human fetal liver. Stem Cells Dev 2011; 20: 1247-1257.
18. Pietrosi G, Vizzini G, Gerlach J, et al. Phases I-II matched case-control study of human fetal liver cell transplantation for treatment of chronic liver disease. Cell Transplant 2015; 24: 1627-1638.

19. Habibullah CM, Syed IH, Qamar A, et al. Human fetal hepatocyte transplantation in patients with fulminant hepatic failure. Transplantation 1994; 58: 951-952.

20. Khan AA, Shaik MV, Parveen N, et al. Human fetal liver-derived stem cell transplantation as supportive modality in the management of end-stage decompensated liver cirrhosis. Cell Transplant 2010; 19: 409-418.

21. Cardinale V, Carpino G, Gentile R, et al. Transplantation of human fetal biliary tree stem/progenitor cells into two patients with advanced liver cirrhosis. BMC Gastroenterol 2014; 14: 204.

22. Schmelzer E. Hepatic Progenitors of the liver and extra-hepatic tissues. In: Baptista P (Ed.). Liver regeneration. InTech 2012; 43-62.

23. Osta WA, Chen Y, Mikhitarian K, et al. EpCAM is overexpressed in breast cancer and is a potential target for breast cancer gene therapy. Cancer Res 2004; 64: 5818-5824.

24. Yanamoto S, Kawasaki G, Yoshitomi I, et al. Clinicopathologic significance of EpCAM expression in squamous cell carcinoma of the tongue and its possibility as a potential target for tongue cancer gene therapy. Oral Oncol 2007; $3: 3$.

25. Maghzal N, Kayali HA, Rohani N, et al. EpCAM controls actomyosin contractility and cell adhesion by direct inhibition of PKC. Dev Cell 2013; 27: 263-277.

26. Maetzel D, Denzel S, Mack B, et al. Nuclear signalling by tumour-associated antigen EpCAM. Nat Cell Biol 2009; 11: 162-171.

27. Gerlach JC, Foka HG, Thompson RL, et al. Epithelial cell adhesion molecule fragments and signaling in primary human liver cells. J Cell Physiol 2018; 233: 4841-4851.

28. Lazaro CA, Croager EJ, Mitchell C, et al. Establishment, characterization, and long-term maintenance of cultures of human fetal hepatocytes. Hepatology 2003; 38: 1095-1106.

29. Huch M, Dollé L. The plastic cellular states of liver cells: Are EpCAM and Lgr5 fit for purpose? Hepatology 2016; 64: 652-662. 